\title{
MANIFOLDS OF HOLOMORPHIC MAPPINGS FROM STRONGLY PSEUDOCONVEX DOMAINS*
}

\author{
FRANC FORSTNERIČ ${ }^{\dagger}$ \\ Dedicated to Professor Salah Baouendi on the occasion of his seventieth birthday
}

\begin{abstract}
Let $D$ be a bounded strongly pseudoconvex domain in a Stein manifold, and let $Y$ be a complex manifold. We show that many classical spaces of maps $\bar{D} \rightarrow Y$ which are holomorphic in $D$ are infinite dimensional complex manifolds which are modeled on locally convex topological vector spaces (Banach, Hilbert or Fréchet). This holds in particular for Hölder and Sobolev spaces of holomorphic maps.
\end{abstract} pings

Key words. Stein manifolds, strongly pseudoconvex domains, manifolds of holomorphic map-

AMS subject classifications. 32E10, 32E30, 32H02, 46G20, 46T10, 54C35, 58B12, 58D15

1. Introduction. Given complex manifolds (or complex spaces) $X$ and $Y$, it is a natural question whether the space $\mathcal{H}(X, Y)$ of all holomorphic mappings $X \rightarrow Y$ is also a complex manifold (resp. a complex space). If $X$ is a compact complex space without boundary then $\mathcal{H}(X, Y)$ is a finite dimensional complex space which can be identified with an open subset in the Douady space $\mathcal{D}(X \times Y)$, [2, Theorem 1.5]. The set of all holomorphic maps from a noncompact manifold in general does not admit any particularly nice structure.

In $\S 2$ of this paper we prove the following results.

TheOREM 1.1. Let $D$ be a relatively compact strongly pseudoconvex domain in a Stein manifold, and let $Y$ be a complex manifold.

(i) The Hölder space $\mathcal{A}^{k, \alpha}(D, Y)=\mathcal{C}^{k, \alpha}(\bar{D}, Y) \cap \mathcal{H}(D, Y)$ is a complex Banach manifold for every $k \in \mathbb{Z}_{+}$and $0 \leq \alpha<1$.

(ii) $\mathcal{A}^{\infty}(D, Y)=\mathcal{C}^{\infty}(\bar{D}, Y) \cap \mathcal{H}(D, Y)$ is a complex Fréchet manifold.

(iii) The Sobolev space $L_{\mathcal{O}}^{k, p}(D, Y)=L^{k, p}(\bar{D}, Y) \cap \mathcal{H}(D, Y)$ is a complex Banach manifold for $k \in \mathbb{N}, p \geq 1$ and $k p>\operatorname{dim}_{\mathbb{R}} D$ (resp. a complex Hilbert manifold if $p=2$ ).

If $L(D, Y)$ denotes any of the above manifolds of maps then the tangent space $T_{f} L(D, Y)$ at a point $f \in L(D, Y)$ is $L_{h}\left(D, f^{*} T Y\right)$, the space of sections of class $L(D)$ of the complex vector bundle $h: f^{*} T Y \rightarrow \bar{D}$. If $D$ is contractible, or if $\operatorname{dim} D=1$, then $T_{f} L(D, Y) \approx L\left(D, \mathbb{C}^{m}\right)$ with $m=\operatorname{dim} Y$.

The analogous conclusions hold if $\bar{D}$ is a compact complex manifold with Stein interior $D$ and smooth strongly pseudoconvex boundary $b D$; according to Heunemann [21] and Ohsawa [31] (see also Catlin [4]) such $\bar{D}$ embeds as a smoothly bounded strongly pseudoconvex domain in a Stein manifold.

The special case of Theorem 1.1 (i) with $\alpha=0$ was proved recently in [11].

\footnotetext{
${ }^{*}$ Received September 24, 2006; accepted for publication February 2, 2007.

$\dagger$ Institute of Mathematics, Physics and Mechanics, University of Ljubljana, Jadranska 19, 1000 Ljubljana, Slovenia (franc.forstneric@fmf.uni-lj.si). Research supported by grants P1-0291 and J16173, Republic of Slovenia.
} 
Before proceeding we recall some of the known results on this subject. If $M$ is a compact smooth manifold, possibly with boundary, and $Y$ is a smooth manifold without boundary then the space of Hölder maps $\mathcal{C}^{k, \alpha}(M, Y)\left(k \in \mathbb{Z}_{+}, 0 \leq \alpha<1\right)$, the space of Sobolev maps $L^{k, p}(M, Y)(k \in \mathbb{N}, 1 \leq p<\infty, k p>\operatorname{dim} M)$, as well as many other natural mapping spaces, are infinite dimensional Banach manifolds (Fréchet if we consider $\mathcal{C}^{\infty}$ maps). For results in this direction (also for sections of smooth fiber bundles over compact smooth manifolds) see Palais [32, 33], Elıasson [12], Krikorian [25], Penot [34], Riddell [35]; for noncompact source manifolds see also Cantor [3]. The cited works deal with important foundational questions of nonlinear global analysis and provide the basic framework for the study of global partial differential operators and the calculus of variations.

These results do not apply directly in the holomorphic category, the main reason being that it is much more difficult (and in general impossible) to find holomorphic tubular neighborhoods. Besides the already mentioned work of Douady and others on compact complex subspaces of a complex space, we are only aware of a few scattered results. Ivashkovich and Shevchishin considered certain manifolds of pseudoholomorphic maps from Riemann surfaces to almost complex manifolds [23, 24]. Quite recently, Lempert [29] proved that for a compact smooth manifold $M$ and a complex manifold $Y$ the space $\mathcal{C}^{k}(M, Y)$ is an infinite dimensional complex Banach manifold (Fréchet if $k=\infty$ ). It is expected that, like their real counterparts, these generalized loop spaces could be useful in the study of geometric properties of the target manifold.

Our proof of Theorem 1.1 is based on the following result of possible independent interest, concerning the existence of tubular Stein neighborhoods of holomorphic graphs with continuous boundary values over strongly pseudoconvex Stein domains.

TheOrem 1.2. Assume that $h: X \rightarrow S$ is a holomorphic submersion of a complex manifold $X$ onto a Stein manifold $S, D \Subset S$ is a strongly pseudoconvex domain with $\mathcal{C}^{2}$ boundary in $S$, and $f: \bar{D} \rightarrow X$ is a continuous section of $h$ which is holomorphic in $D$.

There exists a holomorphic vector bundle $\pi: E \rightarrow U$ over an open set $U \subset S$ containing $\bar{D}$, and for every open set $\Omega_{0} \subset X$ containing $f(\bar{D})$ there exist a Stein open set $\Omega$ in $X$ with $f(\bar{D}) \subset \Omega \subset \Omega_{0}$ and a biholomorphic map $\Theta: \Omega \rightarrow \Theta(\Omega) \subset E$ which maps the fiber $\Omega_{z}=h^{-1}(z) \cap \Omega$ over any point $z \in h(\Omega) \subset U$ biholomorphically onto an open convex set $\widetilde{\Omega}_{z}=\Theta\left(\Omega_{z}\right)$ in the fiber $E_{z}=\pi^{-1}(z)$.

If $\Theta$ is as in Theorem 1.2 then the map $g \rightarrow \Theta \circ g$ induces an isomorphism between the space of sections of the restricted submersion $X_{\bar{D}}=h^{-1}(\bar{D}) \rightarrow \bar{D}$ which are sufficiently uniformly close to $f$, and the space of sections of the complex vector bundle $E_{\bar{D}} \rightarrow \bar{D}$ which are close to $\Theta \circ f$. For each of the mapping spaces in Theorem 1.1 this provides a local holomorphic chart around an element $f$, and it is easily seen that such charts are holomorphically compatible and hence define a complex manifold structure on the relevant space of sections of $X_{\bar{D}} \rightarrow \bar{D}$. Further details of the proof of Theorem 1.1 are given in $\S 2$.

In the special case when the section $f$ in Theorem 1.2 extends holomorphically to a neighborhood of $\bar{D}$, the conclusion of Theorem 1.2 follows by combining Siu's theorem [37] (or Schneider's theorem [36]) with the Docquier-Grauert tubular neighborhood theorem [9]; in this case $\Theta$ can be chosen to map $f$ onto the zero section of $E$.

The following is an immediate corollary to Theorem 1.2. Examples in $\S 5$ show that the conclusion fails in general for images of holomorphic maps. 
Corollary 1.3. If $D \Subset S$ are as in Theorem 1.2, $Y$ is a complex manifold and $f: \bar{D} \rightarrow Y$ is a continuous map which is holomorphic in $D$ then the graph $G_{f}=$ $\{(z, f(z)): z \in \bar{D}\}$ admits a basis of open Stein neighborhoods in $S \times Y$.

There are at least two possible ways to prove Theorem 1.2. One approach is by plurisubharmonic functions and Grauert's characterization of Stein manifolds [16, Theorem 2] (I wish to thank the referee for indicating this possibility). To this end one needs

(i) a strongly plurisubharmonic function $\rho$ in a neighborhood of the compact set $f(\bar{D})$ in $X$, and

(ii) a nonnegative (weakly) plurisubharmonic function $\tau$ in a neighborhood of $f(\bar{D})$ which vanishes precisely on $f(\bar{D})$.

For small enough $\epsilon>0$ the function $\rho+\frac{1}{\epsilon-\tau}$ is then a strongly plurisubharmonic exhaustion function on $\Omega_{\epsilon}=\{\tau<\epsilon\} \subset X$ and hence $\Omega_{\epsilon}$ is Stein. Functions $\rho$ and $\tau$ with these properties can be found by classical methods.

We choose to present a different proof which is based on the method of holomorphic sprays developed in $[10,11]$. This method explicitly produces a holomorphic vector bundle $\pi: E \rightarrow U$ as in Theorem 1.1 whose total space $E$ contains a biholomorphic copy of a Stein neighborhood of $f(\bar{D})$. We give a brief outline; for the details see Sec. $\S 4$.

In [11] the authors constructed a dominating spray with a given central section, holomorphic over $D$ and continuous over $\bar{D}$, by successively gluing sprays over small subsets of $\bar{D}$. The key ingredient of this construction is a Cartan type splitting lemma with control up to the boundary [10, Theorem 3.2]; its proof uses a sup-norm bounded linear solution operator to the $\bar{\partial}$-equation for $(0,1)$-forms on strongly pseudoconvex domains. Here we work with fiberwise injective holomorphic sprays defined on (not necessarily trivial) holomorphic vector bundles of class $\mathcal{A}(D)$. To a spray over $\bar{D}$ we attach finitely many additional sprays over small open sets in $S$ whose union covers the boundary of $D$. By improving the splitting lemma (Lemma 3.2 below) we insure that $f(\bar{D})$ is contained in the range of the resulting holomorphic spray; the inverse of this spray is the map $\Theta$ in Theorem 1.2. We give a proof of Lemma 3.2 based on the implicit function theorem in Banach spaces, simpler than the one in [10] where iteration was used.

For the general theory of Stein manifolds we refer to [17] and [22]; for real analysis in infinite dimensions see [32], and for complex analysis in infinite dimensions see [8] and [28].

2. Complex manifolds of holomorphic maps. Let $D$ be a relatively compact domain with piecewise $\mathcal{C}^{1}$ boundary in $\mathbb{C}^{n}$. We consider the following function spaces on $D$ :

(i) For $k \in \mathbb{Z}_{+}$and $0 \leq \alpha<1, \mathcal{A}^{k, \alpha}(D)$ is the Banach space of all functions $\bar{D} \rightarrow \mathbb{C}$ in the Hölder class $\mathcal{C}^{k, \alpha}(\bar{D})$ which are holomorphic in $D$. When $\alpha=0$ we shall write $\mathcal{A}^{k, 0}=\mathcal{A}^{k}$ and $\mathcal{A}^{0}=\mathcal{A}$.

(ii) $\mathcal{A}^{\infty}(D)=\cap_{k=0}^{\infty} \mathcal{A}^{k}(D)$ is the Fréchet space consisting of all $\mathcal{C}^{\infty}$ function $\bar{D} \rightarrow \mathbb{C}$ which are holomorphic in $D$.

(iii) For $k \in \mathbb{Z}_{+}$and $p \geq 1, L_{\mathcal{O}}^{k, p}(D)$ is the Banach space (Hilbert if $p=2$ ) consisting of all holomorphic functions $D \rightarrow \mathbb{C}$ whose partial derivatives of order $\leq k$ belong to $L^{p}(D)$ (with respect to the Lebesgue measure). These are Sobolev spaces of holomorphic functions on $D$. 
If $L(D)$ is any of the above function spaces, we denote by $L\left(D, \mathbb{C}^{m}\right)$ the locally convex topological vector space consisting of maps whose components belong to $L(D)$. In the case (iii) we shall assume $k p>2 n$, so the Sobolev embedding theorem provides a continuous (even compact) inclusion map $L_{\mathcal{O}}^{k, p}(D) \hookrightarrow \mathcal{A}(D)$ (see e.g. Calderon [1]).

Assume now that $D$ is a relatively compact domain with piecewise $\mathcal{C}^{1}$ boundary in an $n$-dimensional complex manifold $S$. Given a complex manifold $Y$ of dimension $m$ without boundary, one can define the mapping space $L(D, Y)$ as follows. (See Lempert $[29, \S 2]$ for the case when $\bar{D}$ is a compact smooth manifold and we are considering the space $\mathcal{C}^{k}(\bar{D}, Y), k \in \mathbb{Z}_{+} \cup\{\infty\}$. For the smooth manifold structure on certain spaces of smooth maps see Palais [32], as well as Cantor [3], Elıasson [12], Krikorian [25], Penot [34], Riddell [35].) Fix a continuous map $f: \bar{D} \rightarrow Y$. Choose finitely many holomorphic coordinates systems $\phi_{j}: U_{j} \rightarrow \widetilde{U}_{j} \subset \mathbb{C}^{n}$ on $S$, and $\psi_{j}: W_{j} \rightarrow \widetilde{W}_{j} \subset \mathbb{C}^{m}$ on $Y$, such that $f\left(\bar{D} \cap U_{j}\right) \subset W_{j}$ for all $j$. Also choose open subsets $V_{j} \Subset U_{j}$ such that $\bar{D} \subset \cup_{j} V_{j}$ and $V_{j} \cap D$ has piecewise $\mathcal{C}^{1}$ boundary for each $j$. Then $f \in L(D, Y)$ precisely when for each $j$ the restriction $f_{j}$ of the map $\psi_{j} \circ f \circ \phi_{j}^{-1}$ to the set $\phi_{j}\left(\overline{D \cap V_{j}}\right) \Subset \widetilde{U}_{j}$ belongs to $L\left(\phi_{j}\left(D \cap V_{j}\right), \mathbb{C}^{m}\right)$; the definition is independent of the choices of charts. Further, given an open neighborhood $\mathcal{U}_{j} \subset L\left(\phi_{j}\left(D \cap V_{j}\right), \mathbb{C}^{m}\right)$ of $f_{j}$ for every $j$, the corresponding neighborhood of $f$ in $L(D, Y)$ consists of all maps $g: \bar{D} \rightarrow Y$ such that $g\left(\overline{D \cap V_{j}}\right) \subset W_{j}$ and the restriction $g_{j}$ of $\psi_{j} \circ g \circ \phi_{j}^{-1}$ to $\phi_{j}\left(\overline{D \cap V_{j}}\right)$ belongs to $\mathcal{U}_{j}$ for all $j$.

Proof of Theorem 1.1. Let $L(D, Y)$ denote any one of the above spaces; observe that it is a subset of $\mathcal{A}(D, Y)$. We need to construct holomorphic charts in $L(D, Y)$.

Given a holomorphic vector bundle $\pi: E \rightarrow U$ over an open set $U \subset S$ containing $\bar{D}$, we denote by $L_{h}(D, E)$ the space of all section $\bar{D} \rightarrow E_{\bar{D}}$ of $h$ over $\bar{D}$ which belong to $L(D, E)$. This is a locally convex topological vector space; Banach for $\mathcal{A}^{k, \alpha}$ or $L_{\mathcal{O}}^{k, p}$, Hilbert for $L_{\mathcal{O}}^{k, 2}$, and Fréchet for $\mathcal{A}^{\infty}$.

Fix a map $f \in L(D, Y)$; so $f$ is continuous on $\bar{D}$ and holomorphic on $D$. Theorem 1.2 furnishes an open Stein neighborhood $\Omega \subset S \times Y$ of the graph $G_{f}=\{(z, f(z)): z \in$ $\bar{D}\}$ and a biholomorphic map $\Theta: \Omega \rightarrow \widetilde{\Omega} \subset E$ onto an open set $\widetilde{\Omega}$ in the total space of a holomorphic vector bundle $\pi: E \rightarrow U$ such that $\bar{D} \subset U \subset S$ and $\pi \circ \Theta: \Omega \rightarrow S$ is the restriction to $\Omega$ of the base projection $(z, y) \rightarrow z$. Since $\Theta$ is holomorphic in a neighborhood of $G_{f}$, the map

$$
\bar{D} \ni z \rightarrow \theta(f)(z):=\Theta(z, f(z)) \in E_{z}
$$

is a section of the restricted bundle $E_{\bar{D}} \rightarrow \bar{D}$ which belongs to the space $L_{h}(D, E)$. The graph $G_{g}$ of any $g \in L(D, Y)$ sufficiently near $f$ is also contained in $\Omega$, and the composition with $\Theta$ defines an isomorphism $g \rightarrow \theta(g)=\Theta(\cdot, g)$ between an open neighborhood of $f$ in $L(D, Y)$ and an open neighborhood of $\theta(f)$ in $L_{h}(D, E)$; we take $\theta$ as a Banach (or Fréchet) chart on $L(D, Y)$. It is easily verified that the transition map between any such pair of charts is holomorphic (the argument given in [11] for $\mathcal{A}(D, Y)$ applies in all cases; for the Sobolev classes see [33, Theorem 9.10]). The collection of all such charts defines a holomorphic Banach (resp. Fréchet) manifold structure on $L(D, Y)$.

The above construction also shows that the tangent space to the manifold $L(D, Y)$ at a point $f \in L(D, Y)$ can be identified with $L_{h}\left(D, f^{*} T Y\right)$, the space of sections of class $L(D)$ of the complex vector bundle $h: f^{*} T Y \rightarrow \bar{D}$ (the pull-back to $\bar{D}$ of the tangent bundle $T Y$ by the map $f$ ). By the Oka-Grauert principle, homotopic 
maps induce isomorphic pull-back bundles (see Leiterer [26] and Heunemann [19] for the relevant 'up to the boundary' version), and hence $T_{f} L(D, Y)$ is independent of a point $f$ in a connected component of $L(D, Y)$ (up to a complex Banach space isomorphism). Maps belonging to different connected components of $L(D, Y)$ may induce nonisomorphic bundles.

If $D$ is contractible, or if $\operatorname{dim} D=1$ (which means that $D$ is a bordered Riemann surface) then every $\mathcal{A}(D)$-vector bundle over $\bar{D}$ is trivial; in this case $T_{f} L(D, Y) \approx$ $L\left(D, \mathbb{C}^{m}\right)$ with $m=\operatorname{dim} Y$ for every $f \in L(D, Y)$.

The above construction is essentially the same as the one of Palais in the $\mathcal{C}^{\infty}$ case [32, Ch. 13]. With some additional work it might be possible to introduce the categorical (axiomatic) approach as in [32] and thereby extend the result to a wider class of function spaces. Observe that the above proof actually gives the following more general result.

TheOREm 2.1. Assume that $h: X \rightarrow S$ is a holomorphic submersion of a complex manifold $X$ onto a Stein manifold $S$ and $D \Subset S$ is a strongly pseudoconvex domain with $\mathcal{C}^{2}$ boundary in $S$. Let $L$ be any of the classes $\mathcal{A}^{k, \alpha}, \mathcal{A}^{\infty}$ or $L_{\mathcal{O}}^{k, p}\left(k p>\operatorname{dim}_{\mathbb{R}} S\right)$. Then the space of sections

$$
L_{h}(D, X)=\{f \in L(D, X): h(f(z))=z, z \in \bar{D}\}
$$

is a complex Banach manifold (Fréchet for $L=\mathcal{A}^{\infty}$ ).

REMARK 2.2. The method in [11] can be used to obtain the same result in the more general case when $h: X \rightarrow \bar{D}$ is a smooth submersion onto $\bar{D}$ which is holomorphic over $D$; an example is a smooth fiber bundle over $\bar{D}$ which is holomorphic over $D$. However, for holomorphic submersions which extend holomorphically to a neighborhood of $\bar{D}$ in $S$, the above construction is simpler than the one in [11]. Indeed, we do not need a new splitting lemma for each of the function spaces - we only need it for the space $\mathcal{A}_{h}(D, E)$ where $h: E \rightarrow \bar{D}$ is a complex vector bundle which is holomorphic over $D$.

The complex structures introduced above enjoy the following functorial property; compare with [29, Proposition 2.3] and observe that the proof given there carries over to our situation as well.

Proposition 2.3. Let $D \Subset S$ be a strongly pseudoconvex domain in a Stein manifold $S$, let $Y$ and $Y^{\prime}$ be complex manifolds, and let $\Phi: S \times Y \rightarrow Y^{\prime}$ be a holomorphic map. Then the induced map $\Phi_{*}: L(D, Y) \rightarrow L\left(D, Y^{\prime}\right)$ defined by $\Phi_{*}(f)(z)=\Phi(z, f(z))(z \in \bar{D})$ is holomorphic.

The discussion following Proposition 2.3 in [29] also shows that the functorial property described in the above proposition characterizes the complex structures under consideration.

These complex structures are functorial also with respect to maps of the source domains: Given strongly pseudoconvex domains $D \Subset S, D^{\prime} \Subset S^{\prime}$ in Stein manifolds $S$ resp. $S^{\prime}$, and given a holomorphic map $\Psi: S \rightarrow S^{\prime}$ satisfying $\Psi(D) \subset D^{\prime}$, the pullback map $\Psi^{*}: L\left(D^{\prime}, Y\right) \rightarrow L(D, Y)$ defined by $f \rightarrow f \circ \Psi$ is holomorphic (see [29, Proposition 2.4]). In particular, the evaluation map $e: \bar{D} \times L(D, Y) \rightarrow Y, e(z, f)=$ $f(z)$, is continuous, and is holomorphic in $f$ for a fixed $z \in \bar{D}$ (see the proof of Proposition 2.5 in [29]). Weaker hypothesis on $\Psi$ may suffice in individual cases. 
3. A splitting lemma. In this section we prove a splitting lemma for fiberwise injective holomorphic maps on holomorphic vector bundles with continuous boundary values. Lemma 3.2 below is the main ingredient in the proof of Theorem 1.2. Although it is a minor extension of Theorem 3.2 in [10] (which applies to trivial bundles), we give a simpler proof based on the implicit function theorem.

Definition 3.1. [11, Def. 2.3] A pair of open subsets $D_{0}, D_{1} \Subset S$ in a Stein manifold $S$ is said to be a Cartan pair of class $\mathcal{C}^{\ell}(\ell \geq 2)$ if

(i) $D_{0}, D_{1}, D=D_{0} \cup D_{1}$ and $D_{0,1}=D_{0} \cap D_{1}$ are strongly pseudoconvex domains with $\mathcal{C}^{\ell}$ boundaries, and

(ii) $\overline{D_{0} \backslash D_{1}} \cap \overline{D_{1} \backslash D_{0}}=\emptyset$ (the separation property).

$D_{1}$ is a convex bump on $D_{0}$ if in addition there is a biholomorphic map from an open neighborhood of $\bar{D}_{1}$ in $S$ onto an open subset of $\mathbb{C}^{n}(n=\operatorname{dim} S)$ which maps $D_{1}$ and $D_{0,1}$ onto strongly convex domains in $\mathbb{C}^{n}$.

Suppose that $D \Subset S$ is a strongly pseudoconvex domain in a Stein manifold $S$ and $\pi: E \rightarrow \bar{D}$ is a continuous complex vector bundle which is holomorphic over $D$ (an $\mathcal{A}(D)$-vector bundle). Such $E$ embeds as an $\mathcal{A}(D)$-vector subbundle $E^{\prime}$ of a trivial bundle $\mathbb{T}^{N}=\bar{D} \times \mathbb{C}^{N}$ for a sufficiently large integer $N$, and for every such embedding there is a Whitney direct sum decomposition $\mathbb{T}^{N}=E^{\prime} \oplus E^{\prime \prime}$ of class $\mathcal{A}(D)$. (These facts follows from Cartan's Theorem B for $\mathcal{A}(D)$-vector bundles; see Leiterer $[26,27]$ and Heunemann $[20,21]$.) Fix such a decomposition and identify $E$ with $E^{\prime}$. We shall denote the variable in $\bar{D}$ by $z$ and the variable in $\mathbb{C}^{N}$ by $t$. On $\{z\} \times \mathbb{C}^{N}$ we have a unique decomposition $t=t^{\prime} \oplus t^{\prime \prime} \in E_{z}^{\prime} \oplus E_{z}^{\prime \prime}$ which is of class $\mathcal{A}(D)$ with respect to $z \in \bar{D}$. Let $|\cdot|$ denote the standard Euclidean norm on $\mathbb{C}^{N}$, and let $\mathbb{B}=\left\{t \in \mathbb{C}^{N}:|t|<1\right\}$. For every $r>0$ and $z \in \bar{D}$ set

$$
E_{z, r}=\left\{t=t^{\prime} \oplus 0^{\prime \prime} \in E_{z}:|t|<r\right\}=E_{z} \cap r \mathbb{B} .
$$

Given a subset $K \subset \bar{D}$ and $r>0$ we shall write

$$
E_{K}=\cup_{z \in K} E_{z}, \quad E_{K, r}=\cup_{z \in K} E_{z, r}=E_{K} \cap(K \times r \mathbb{B}) .
$$

Every continuous fiber-preserving map $E_{K, r} \rightarrow E_{K}$ is of the form $\gamma\left(z, t^{\prime}\right)=\left(z, \psi\left(z, t^{\prime}\right)\right)$ for $z \in K$ and $\left|t^{\prime}\right|=\left|t^{\prime} \oplus 0^{\prime \prime}\right|<r$; we shall say that $\gamma$ is of class $\mathcal{A}$ if it is holomorphic in the interior of its domain. Let $i d\left(z, t^{\prime}\right)=\left(z, t^{\prime}\right)$ denote the identity map on $E$. Set

$$
\|\gamma-i d\|_{K, r}=\sup \left\{\left|\psi\left(z, t^{\prime}\right)-t^{\prime}\right|: z \in K,\left|t^{\prime}\right|<r\right\} .
$$

Lemma 3.2. Let $D=D_{0} \cup D_{1}$ be a Cartan pair of class $\mathcal{C}^{2}$ in a Stein manifold $S$, and let $\pi: E \rightarrow \bar{D}$ be an $\mathcal{A}(D)$-bundle. Set $K=\bar{D}_{0,1}=\bar{D}_{0} \cap \bar{D}_{1}$. Given numbers $0<r^{\prime}<r$ and $\epsilon>0$, there is a number $\delta>0$ satisfying the following. For every fiber preserving map $\gamma: E_{K, r} \rightarrow E_{K}$ of class $\mathcal{A}\left(E_{K, r}\right)$ with $\|\gamma-i d\|_{K, r}<\delta$ there exist injective fiber preserving maps $\alpha: E_{\bar{D}_{0}, r^{\prime}} \rightarrow E_{\bar{D}_{0}}, \beta: E_{\bar{D}_{1}, r^{\prime}} \rightarrow E_{\bar{D}_{1}}$, of class $\mathcal{A}$ on their respective domains, satisfying $\|\alpha-i d\|_{\bar{D}_{0}, r}<\epsilon,\|\beta-i d\|_{\bar{D}_{1}, r}<\epsilon$ and

$$
\gamma \circ \alpha=\beta \quad \text { on } E_{K, r^{\prime}} .
$$

If in addition $\gamma$ preserves the zero section (i.e., $\gamma(z, 0)=(z, 0)$ for $z \in K$ ) then $\alpha$ and $\beta$ can be chosen to satisfy the same property. 
If the Cartan pair $D=D_{0} \cup D_{1}$ is of class $\mathcal{C}^{\ell}, \ell \geq 2$, then for any integer $l \in\{0,1, \ldots, \ell\}$ the analogous result holds with the bundle $E$ and the maps $\alpha, \beta, \gamma$ of class $\mathcal{A}^{l}$ on their respective domains (i.e., holomorphic inside and of class $\mathcal{C}^{l}$ up to the boundary), with $\mathcal{C}^{l}$ estimates.

Proof. We begin with the special case when the bundle $E$ is trivial, $E=\mathbb{T}^{N}=$ $\bar{D} \times \mathbb{C}^{N}$ for some $N \in \mathbb{N}$. Although in this case the result coincides with Theorem 3.2 in [10], we give a new proof based on the implicit function theorem. (It is similar to the proof of Proposition 5.2 in [13, p. 141].)

Recall that $\left(\gamma(z, t)=(z, \psi(z, t))\right.$, where $\psi: K \times r \mathbb{B} \rightarrow \mathbb{C}^{N}$ is close to the map $\psi_{0}(z, t)=t$. We denote by $C_{r}$ (resp. by $\Gamma_{r}$ ) the Banach space consisting of all continuous maps $K \times r \mathbb{B} \ni(z, t) \rightarrow \psi(z, t) \in \mathbb{C}^{N}$ which are holomorphic in the interior $D_{0,1} \times r \mathbb{B}$ of $K \times r \mathbb{B}$ and satisfy

$$
\begin{aligned}
& \|\psi\|_{C_{r}}=\sup _{(z, t) \in K \times r \mathbb{B}}|\psi(z, t)|<+\infty, \\
& \|\psi\|_{\Gamma_{r}}=\sup _{(z, t) \in K \times r \mathbb{B}}\left(|\psi(z, t)|+\left|\partial_{t} \psi(z, t)\right|\right)<+\infty .
\end{aligned}
$$

Here, $\partial_{t}$ denotes the partial differential with respect to the variable $t \in \mathbb{C}^{N}$, and $\left|\partial_{t} \psi(z, t)\right|$ is the Euclidean operator norm.

Replacing the number $r>0$ in Lemma 3.2 with a slightly smaller number we can assume (in view of the Cauchy estimates) that $\psi$ belongs to $\Gamma_{r}$ and that $\left\|\psi-\psi_{0}\right\|_{\Gamma_{r}}$ is as small as desired, where $\psi_{0}(z, t)=t$. Fix such $r$ and choose a number $r^{\prime}$ with $0<r^{\prime}<r$. Let $A_{r^{\prime}}$ (resp. by $B_{r^{\prime}}$ ) denote the Banach space of all continuous maps $\bar{D}_{0} \times r^{\prime} \mathbb{B} \rightarrow \mathbb{C}^{N}$ (resp. $\bar{D}_{1} \times r^{\prime} \mathbb{B} \rightarrow \mathbb{C}^{N}$ ) which are holomorphic in the interior of the respective set, endowed with the sup-norm. By [10, Lemma 3.4] there exist continuous linear operators $\mathcal{A}: C_{r^{\prime}} \rightarrow A_{r^{\prime}}, \mathcal{B}: C_{r^{\prime}} \rightarrow B_{r^{\prime}}$ satisfying

$$
c=\mathcal{A}(c)-\mathcal{B}(c), \quad c \in C_{r^{\prime}} .
$$

The proof in [10] uses a linear solution operator for the $\bar{\partial}$-equation on the level of $(0,1)$-forms on $D$ satisfying sup-norm estimates, and the variables $t$ are treated as parameters.

Given $\psi \in \Gamma_{r}$ sufficiently near $\psi_{0}$ and $c \in C_{r^{\prime}}$ near 0 , we define

$$
\Phi(\psi, c)(z, t)=\psi(z, t+\mathcal{A}(c)(z, t))-(t+\mathcal{B}(c)(z, t)), \quad(z, t) \in K \times r^{\prime} \mathbb{B} .
$$

It is easily verified that $(\psi, c) \rightarrow \Phi(\psi, c)$ is a $\mathcal{C}^{1}$ (even smooth) map from an open neighborhood of the point $\left(\psi_{0}, 0\right)$ in the Banach space $\Gamma_{r} \times C_{r^{\prime}}$ to the Banach space $C_{r^{\prime}}$. (Although we are composing maps which are only continuous up to the boundary of their respective domains in the $z$-variable, we are inserting $\mathcal{A}(c)$ in the second variable of $\psi$, and $\psi$ is holomorphic with respect to that variable on a larger domain.)

Since $\Phi\left(\psi_{0}, c\right)=\mathcal{A}(c)-\mathcal{B}(c)=c$ by (3.2), the implicit function theorem shows that in a neighborhood of $\left(\psi_{0}, 0\right)$ in $\Gamma_{r} \times C_{r^{\prime}}$ we can solve the equation $\Phi(\psi, c)=0$ on $c$; that is, there is a $\mathcal{C}^{1}$ map $\psi \rightarrow \mathcal{C}(\psi) \in C_{r^{\prime}}$, defined in an open neighborhood of $\psi_{0}$ in $\Gamma_{r}$ and satisfying

$$
\Phi(\psi, \mathcal{C}(\psi))=0, \quad \mathcal{C}\left(\psi_{0}\right)=0
$$

Consider the functions

$$
a_{\psi}=t+\mathcal{A} \circ \mathcal{C}(\psi) \in A_{r^{\prime}}, \quad b_{\psi}=t+\mathcal{B} \circ \mathcal{C}(\psi) \in B_{r^{\prime}} .
$$


From (3.3) and the definition of $\Phi$ we obtain

$$
\psi\left(z, a_{\psi}(z, t)\right)=b_{\psi}(z, t), \quad(z, t) \in K \times r^{\prime} \mathbb{B} .
$$

Setting $\alpha(z, t)=\left(z, a_{\psi}(z, t)\right), \beta(z, t)=\left(z, b_{\psi}(z, t)\right)$, we see that (3.4) gives $\gamma \circ \alpha=\beta$. We also get sup-norm estimates on $a_{\psi}-\psi_{0}$ (resp. on $b_{\psi}-\psi_{0}$ ) on $\bar{D}_{0} \times r^{\prime} \mathbb{B}$ (resp. on $\left.\bar{D}_{1} \times r^{\prime} \mathbb{B}\right)$ in terms of $\left\|\psi-\psi_{0}\right\|_{\Gamma_{r}}$. If the latter number is sufficiently small, the maps $a_{\psi}$ and $b_{\psi}$ are as close as desired to the map $\psi_{0}(z, t)=t$ and hence, after we shrink $r^{\prime}$ slightly and apply again the Cauchy estimates in the $t$-variable, we can assume that they are fiberwise injective holomorphic. This proves Lemma 3.2 when $E=\bar{D} \times \mathbb{C}^{N}$.

The case with $\mathcal{C}^{l}$ boundary values is obtained in the same way by using the appropriate Banach spaces; compare with Theorem 3.2 in [10].

It remains to consider the general case when $E$ is an $\mathcal{A}(D)$-vector subbundle of $\mathbb{T}^{N}=\bar{D} \times \mathbb{C}^{N}$. As before we identify $E$ with its image $E^{\prime} \subset \mathbb{T}^{N}$ and write $\mathbb{T}^{N}=$ $E^{\prime} \oplus E^{\prime \prime}$ (an $\mathcal{A}(D)$-decomposition). Let $t=t^{\prime} \oplus t^{\prime \prime} \in E_{z}^{\prime} \oplus E_{z}^{\prime \prime}$ denote the corresponding splitting of the fiber variable. We associate to each self-map $\gamma\left(z, t^{\prime}\right)=\left(z, \psi^{\prime}\left(z, t^{\prime}\right)\right)$ of $E^{\prime}$ the self-map

$$
\widetilde{\gamma}(z, t)=(z, \psi(z, t)), \quad \psi(z, t)=\psi^{\prime}\left(z, t^{\prime}\right) \oplus t^{\prime \prime}
$$

of $\mathbb{T}^{N}$ (we added the identity map on the second summand $E^{\prime \prime}$ ). If $\psi^{\prime}$ is sufficiently close to the map $\left(z, t^{\prime}\right) \rightarrow t^{\prime}$ then $\psi$ is close to the map $\psi_{0}(z, t)=t$, and the first part of the Lemma (for the trivial bundle) furnishes $\mathbb{C}^{N}$-valued maps

$$
\begin{array}{ll}
a(z, t)=a^{\prime}(z, t) \oplus a^{\prime \prime}(z, t), & (z, t) \in \bar{D}_{0} \times r^{\prime} \mathbb{B}, \\
b(z, t)=b^{\prime}(z, t) \oplus b^{\prime \prime}(z, t), & (z, t) \in \bar{D}_{1} \times r^{\prime} \mathbb{B}
\end{array}
$$

satisfying $\psi(z, a(z, t))=b(z, t)$ for $(z, t) \in K \times r^{\prime} \mathbb{B}$. Comparing the $E^{\prime}$ and the $E^{\prime \prime}$ components of this identity at $t^{\prime \prime}=0$ we get

$$
\psi^{\prime}\left(z, a^{\prime}\left(z, t^{\prime}\right)\right)=b^{\prime}\left(z, t^{\prime}\right), \quad a^{\prime \prime}\left(z, t^{\prime}\right)=b^{\prime \prime}\left(z, t^{\prime}\right) .
$$

Hence the maps $\alpha\left(z, t^{\prime}\right)=\left(z, a^{\prime}\left(z, t^{\prime}\right)\right)$ and $\beta\left(z, t^{\prime}\right)=\left(z, b^{\prime}\left(z, t^{\prime}\right)\right)$ satisfy the conclusion of Lemma 3.2. $\mathrm{Q}$

4. Proof of Theorem 1.2. Let $f: \bar{D} \rightarrow X$ be a continuous section of a holomorphic submersion $h: X \rightarrow S$ such that $f$ is holomorphic in $D$. Set $\Sigma=f(\bar{D})$. Recall that $V T(X)=\operatorname{ker} d h$ is the vertical tangent bundle of $X$.

By Proposition 4.1 in [11] there exists a map $F: \bar{D} \times r \mathbb{B}^{N} \rightarrow X$ of class $\mathcal{A}\left(D \times r \mathbb{B}^{N}\right)$ for some $r>0$ and $N \in \mathbb{N}$ such that for all $z \in \bar{D}$ we have

(i) $F\left(\{z\} \times r \mathbb{B}^{N}\right) \subset X_{z}=h^{-1}(z)$,

(ii) $F(z, 0)=f(z)$, and

(iii) the map $\left.\partial_{t}\right|_{t=0} F(z, t): \mathbb{C}^{N} \rightarrow V T_{f(z)} X$ is surjective.

A map $F$ with these properties is called a dominating spray of class $\mathcal{A}(D)$ with the central section $F_{0}=F(\cdot, 0)=f$. (We emphasize that the sprays used here are local with respect to the parameter and should not be confused with the global sprays used in the Oka-Grauert theory.)

Set $E_{z}^{\prime \prime}=\left.\operatorname{ker} \partial_{t}\right|_{t=0} F(z, t) \subset \mathbb{C}^{N}(z \in \bar{D})$; this defines an $\mathcal{A}(D)$-subbundle $E^{\prime \prime} \subset$ $\mathbb{T}^{N}:=\bar{D} \times \mathbb{C}^{N}$. By [20] and [27] there exists a complementary $\mathcal{A}(D)$-subbundle $E^{\prime} \subset \mathbb{T}^{N}$ such that $\mathbb{T}^{N}=E^{\prime} \oplus E^{\prime \prime}$. By [19] (see also the Appendix in [10]) we 
can approximate $E^{\prime}$ sufficiently well over $\bar{D}$ by a holomorphic vector subbundle $E \subset$ $U \times \mathbb{C}^{N}$ over an open set $U \subset S$ containing $\bar{D}$ such that $\mathbb{T}^{N}=E_{\bar{D}} \oplus E^{\prime \prime}$.

Let $G$ denote the restriction of $F$ to $E_{\bar{D}, r}=E_{\bar{D}} \cap(K \times r \mathbb{B})$ (3.1). Then $\left.\partial_{t^{\prime}}\right|_{t^{\prime}=0} G\left(z, t^{\prime}\right): E_{z} \rightarrow V T_{f(z)} X$ is a linear isomorphism for every $z \in \bar{D}$, and by decreasing $r>0$ we can insure that $G$ is injective holomorphic on each fiber; such $G$ will be called a fiberwise biholomorphic spray. Note that $E_{\bar{D}}$ is isomorphic to the bundle $\left.V T(X)\right|_{\Sigma}$; when $X=S \times Y$ and $\Sigma$ is the graph of an $\mathcal{A}(D, Y)$-map $f: \bar{D} \rightarrow Y$, the latter bundle is isomorphic to $f^{*} T Y$.

LEMma 4.1. There exist a number $r^{\prime} \in(0, r)$, a decreasing sequence of open sets $O_{1} \supset O_{2} \supset \cdots$ in $S$ with $\cap_{s=1}^{\infty} O_{s}=\bar{D}$, and a sequence of fiberwise biholomorphic sprays $G_{s}: E_{O_{s}, r^{\prime}} \rightarrow X$ such that $G_{s}$ converges to $G$ uniformly on $E_{\bar{D}, r^{\prime}}$ as $s \rightarrow \infty$ and $\Sigma \subset G_{s}\left(E_{O_{s}, r^{\prime}}\right)$ for $s=1,2, \ldots$

Proof of Theorem 1.2. Assume Lemma 4.1 for the moment. Let $\Omega_{0} \subset X$ be an open neighborhood of $f(\bar{D})$. Choose an initial fiberwise biholomorphic spray $G: E_{\bar{D}, r} \rightarrow X$ as above such that $G\left(E_{\bar{D}, r}\right) \subset \Omega_{0}$ (the latter is achieved by decreasing the number $r>0$ if necessary). Let $G_{s}: E_{O_{s}, r^{\prime}} \rightarrow X(s=1,2, \ldots)$ be a sequence of sprays furnished by Lemma 4.1. Set

$$
\widetilde{\Omega}_{s}=E_{O_{s}, r^{\prime}} \subset E, \quad \Omega_{s}=G_{s}\left(E_{O_{s}, r^{\prime}}\right) \subset X, \quad \Theta_{s}=G_{s}^{-1}: \Omega_{s} \rightarrow \widetilde{\Omega}_{s} .
$$

If $s \in \mathbb{N}$ is chosen sufficiently large then $\Omega_{s} \subset \Omega_{0}$, and for such $s$ the map $\Theta_{s}: \Omega_{s} \rightarrow \widetilde{\Omega}_{s}$ satisfies the conclusion of Theorem 1.2.

Proof of Lemma 4.1. We proceed as in the proof of Theorem 5.1 in [11], but paying close attention to the ranges of the approximating sprays in order to insure that each of them contains the graph $\Sigma=f(\bar{D})$.

Since $h: X \rightarrow S$ is a holomorphic submersion, there exist for each point $x_{0} \in X$ open neighborhoods $x_{0} \in W \subset X, h\left(x_{0}\right) \in V \subset S$, and biholomorphic maps $\phi: V \rightarrow$ $\mathbb{B}^{n} \subset \mathbb{C}^{n}, \Phi: W \rightarrow \mathbb{B}^{n} \times \mathbb{B}^{m} \subset \mathbb{C}^{n} \times \mathbb{C}^{m}$, such that $\phi(h(x))=p r_{1}(\Phi(x))$ for every $x \in W$. Note that

$$
\Phi(x)=\left(\phi(h(x)), \phi^{\prime}(x)\right) \in \mathbb{B}^{n} \times \mathbb{B}^{m}, \quad x \in W,
$$

where $\phi^{\prime}=p r_{2} \circ \Phi$. We call such $(W, V, \Phi)$ a special coordinate chart on $X$.

Recall that $G: E_{\bar{D}, r} \rightarrow X$ is a fiberwise biholomorphic spray over $\bar{D}$ with the central section $f$. Narasimhan's lemma on local convexification of a strongly pseudoconvex hypersurface gives finitely many special coordinate charts $\left(W_{j}, V_{j}, \Phi_{j}\right)$ on $X$, with $\Phi_{j}=\left(\phi_{j} \circ h, \phi_{j}^{\prime}\right)$, such that $b D \subset \cup_{j=1}^{j_{0}} V_{j}$ and the following hold for all $j=1, \ldots, j_{0}$ (for (ii) and (iii) we may have to decrease $r>0$ ):

(i) $\phi_{j}\left(b D \cap V_{j}\right)$ is a strongly convex hypersurface in the ball $\mathbb{B}^{n}$,

(ii) the spray $G$ maps $E_{\bar{D} \cap V_{j}, r}$ into $W_{j}$, and

(iii) $\phi_{j}^{\prime} \circ G\left(E_{\bar{D} \cap V_{j}, r}\right) \Subset \mathbb{B}^{m}$.

Fix an $r>0$ such that the above properties hold and choose a number $r^{\prime} \in(0, r)$. Also choose a number $c \in(0,1)$ sufficiently close to 1 such that the open sets $U_{j}=$ $\phi_{j}^{-1}\left(c \mathbb{B}^{n}\right) \Subset V_{j}\left(j=1, \ldots, j_{0}\right)$ still cover $b D$.

By a finite induction we shall find strongly pseudoconvex domains $D=D_{0} \subset$ $D_{1} \subset \cdots \subset D_{j_{0}} \Subset U$, numbers $r=r_{0}>r_{1}>\cdots>r_{j_{0}}=r^{\prime}$ and fiberwise biholomorphic sprays $G_{k}: E_{\bar{D}_{k}, r_{k}} \rightarrow X$ of class $\mathcal{A}\left(k=0,1, \ldots, j_{0}\right)$, with $G_{0}=G$, such that for 
every $k \in\left\{1, \ldots, j_{0}\right\}$ the restriction of $G_{k}$ to $E_{\bar{D}_{k-1}, r_{k}}$ will approximate $G_{k-1}$ in the sup-norm topology. The domain $D_{k}$ will be chosen such that

$$
D_{k-1} \subset D_{k} \subset D_{k-1} \cup V_{k}, \quad b D_{k-1} \cap U_{k} \subset D_{k}
$$

for $k=1, \ldots, j_{0}$; that is, we enlarge (bump out) $D_{k-1}$ inside $V_{k}$ so that the part of $b D_{k-1}$ which lies in the smaller set $U_{k}$ is contained in the next domain $D_{k}$. As the $U_{j}$ 's cover $b D$, the final domain $D_{j_{0}}$ will contain $\bar{D}$ in its interior, and the spray $\widetilde{G}=G_{j_{0}}: E_{\bar{D}_{j_{0}}, r^{\prime}} \rightarrow X$ will approximate $G$ as close as desired on $E_{\bar{D}, r^{\prime}} ;$ in particular, we shall arrange that $\Sigma=f(\bar{D})$ is contained in $\widetilde{G}\left(E_{\bar{D}_{j_{0}}, r^{\prime}}\right)$. To keep the induction going we will also insure at every step that the properties (ii) and (iii) above remain valid with $(D, G)$ replaced by $\left(D_{k}, G_{k}\right)$ for all $k=1, \ldots, j_{0}$. The restriction of $\widetilde{G}$ to the interior $E_{D_{j_{0}}, r^{\prime}}$ can be taken as one of the sprays in the conclusion of the lemma.

The geometric scheme is as in the proof of Theorem 5.1 in [11]. Since all steps are of the same kind, it suffices to explain how to get the pair $\left(D_{1}, G_{1}\right)$ from $(D, G)=$ $\left(D_{0}, G_{0}\right)$. We begin by finding a domain $D_{1}^{\prime} \subset S$ with $\mathcal{C}^{2}$ boundary which is a convex bump on $D=D_{0}$ (Definition 3.1) and such that $\bar{U}_{1} \cap \bar{D} \subset D_{1}^{\prime} \subset V_{1}$. To do this, we shall first find a set $\widetilde{D}_{1}^{\prime} \subset \mathbb{B}^{n}$ with suitable properties and then take $D_{1}^{\prime}=\phi_{1}^{-1}\left(\widetilde{D}_{1}^{\prime}\right)$. Choose a smooth function $\chi \geq 0$ with compact support on $\mathbb{B}^{n}$ such that $\chi=1$ on $c \mathbb{B}^{n}$. Recall that $U_{1}=\phi_{1}^{-1}\left(c \mathbb{B}^{n}\right)$. Let $\tau: \mathbb{B}^{n} \rightarrow \mathbb{R}$ be a strongly convex defining function for the domain $\phi_{1}\left(D \cap V_{1}\right) \subset \mathbb{B}^{n}$. Choose a number $c^{\prime} \in(c, 1)$ close to 1 such that the hypersurface $\phi_{1}\left(b D \cap V_{1}\right)=\{\tau=0\}$ intersects the sphere $\left\{\zeta \in \mathbb{C}^{n}:|\zeta|=c^{\prime}\right\}$ transversely. If $\delta>0$ is sufficiently small then the set

$$
\left\{\zeta \in \mathbb{C}^{n}:|\zeta|<c^{\prime}, \tau(\zeta)<\delta \chi(\zeta)\right\}
$$

could serve our purpose, except that it is not smooth along the intersection of the (convex) hypersurfaces $\left\{|\zeta|=c^{\prime}\right\}$ and $\{\tau=\delta \chi\}$. By rounding off the corners we get a strongly convex set $\widetilde{D}_{1}^{\prime} \subset \mathbb{B}^{n}$ such that $D_{1}^{\prime}=\phi_{1}^{-1}\left(\widetilde{D}_{1}^{\prime}\right) \subset V_{1}$ satisfies the desired properties. (See Fig. 1 which is taken from [11].)

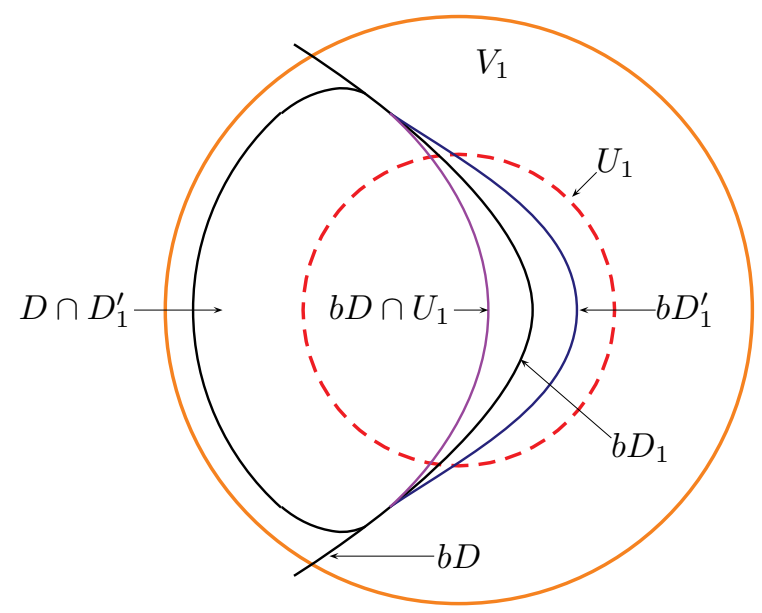

FIG. 1. The domains $D_{1}^{\prime}$ and $D_{1}$

Choose numbers $r_{1}, r_{1}^{\prime}, r_{1}^{\prime \prime}$ with $r^{\prime}<r_{1}<r_{1}^{\prime}<r_{1}^{\prime \prime}<r$. By using the special coordinate chart $\left(W_{1}, V_{1}, \Phi_{1}\right)$ we find an open set $V_{1}^{\prime} \subset V_{1}$ containing $\bar{D} \cap V_{1}$ ( $V_{1}^{\prime}$ will 
depend on the choice of $G^{\prime}$ ) and a fiberwise biholomorphic spray $G^{\prime}: E_{\bar{V}_{1}^{\prime}, r_{1}^{\prime \prime}} \rightarrow X$ with range in $W_{1}$ whose restriction to $E_{\bar{D} \cap \bar{V}_{1}, r_{1}^{\prime \prime}}$ approximates the initial spray $G=G_{0}$ as close as desired in the uniform topology. If the approximation is sufficiently close on $E_{\bar{V}_{1}^{\prime}, r_{1}^{\prime \prime}}$, there exists a (unique) fiberwise biholomorphic map $\gamma: E_{\bar{D}_{\cap} \bar{V}_{1}, r_{1}^{\prime}} \rightarrow E$ of class $\mathcal{A}$ which is close to the identity map and satisfies the equation

$$
G(z, t)=G^{\prime}(\gamma(z, t))=G^{\prime}(z, \psi(z, t)), \quad z \in \bar{D} \cap \bar{V}_{1}, t \in E_{z, r_{1}^{\prime}} .
$$

Applying Lemma 3.2 on the Cartan pair $\left(D, D_{1}^{\prime}\right)$ we obtain $\gamma \circ \alpha=\beta$ on $E_{\bar{D} \cap \bar{V}_{1}, r_{1}}$, where $\alpha: E_{\bar{D}, r_{1}} \rightarrow E$ and $\beta: E_{\bar{D}_{1}^{\prime}, r_{1}} \rightarrow E$ are injective holomorphic maps which are close to the identity on their respective domains. It follows that the fiberwise biholomorphic sprays

$$
G \circ \alpha: E_{\bar{D}, r_{1}} \rightarrow X, \quad G^{\prime} \circ \beta: E_{\bar{D}_{1}^{\prime} \cap \bar{V}_{1}^{\prime}, r_{1}} \rightarrow X
$$

agree on the intersection of their domains, and hence they define a fiberwise biholomorphic spray $G_{1}: E_{\bar{D} \cup\left(\bar{D}_{1}^{\prime} \cap \bar{V}_{1}^{\prime}\right), r_{1}} \rightarrow X$ of class $\mathcal{A}$. By construction $G_{1}$ approximates $G$ uniformly on $E_{\bar{D}, r_{1}}$ as close as desired.

It remains to restrict $G_{1}$ to a suitably chosen strongly pseudoconvex domain $D_{1} \Subset S$ contained in $D \cup\left(D_{1}^{\prime} \cap V_{1}^{\prime}\right)$ and satisfying the other required properties. We choose $D_{1}$ such that it agrees with $D$ outside of $V_{1}$, while

$$
D \cap V_{1}=\phi_{1}^{-1}\left(\left\{\zeta \in \mathbb{B}^{n}: \tau(\zeta)<\epsilon \chi(\zeta)\right\}\right)
$$

for a small $\epsilon>0$ (Fig. 1). By choosing $\epsilon$ sufficiently small (depending on $G_{1}$ ) we can insure that properties (i)-(iii) are satisfied by the pair $\left(D_{1}, G_{1}\right)$.

Applying the same procedure to $\left(D_{1}, G_{1}\right)$ and the special coordinate chart $\left(W_{2}, V_{2}, \Phi_{2}\right)$ we get the next pair $\left(D_{2}, G_{2}\right)$. After $j_{0}$ steps we find a domain $D_{j_{0}} \subset S$ containing $\bar{D}$ and a fiberwise biholomorphic spray $\widetilde{G}=G_{j_{0}}: E_{\bar{D}_{j_{0}}, r^{\prime}} \rightarrow X$ which approximates $G$ as close as desired uniformly on $E_{\bar{D}, r^{\prime}}$. If the approximation is close enough then the range of $\widetilde{G}$ contains $\Sigma$.

The sequence $G_{s}$ in Lemma 4.1 is chosen to consist of sprays $\widetilde{G}$ obtained as above, approximating $G$ ever more closely on $E_{\bar{D}, r^{\prime}}$.

5. Examples and problems. Let $\mathbb{D}$ denote the open unit disc in the complex plane $\mathbb{C}$. Our first example shows that Corollary 1.3 fails in general for maps which are discontinuous at the boundary.

EXAMPLE 5.1. There exists a bounded holomorphic function on $\mathbb{D}$ such that the closure of its graph does not have a Stein neighborhood basis in $\mathbb{C}^{2}$.

Indeed, let $f \in H^{\infty}(\mathbb{D})$ be a bounded holomorphic function on the unit disc such that $\sup _{z \in \mathbb{D}}|f(z)|=1$ and the cluster set of $f$ at every boundary point $e^{i \theta} \in b \mathbb{D}$ equals $\overline{\mathbb{D}}$. (Such functions are easily found by using interpolation theorems for $H^{\infty}(\mathbb{D})$, see [14, Ch. VII].) The closure $K$ of the graph of $f$ in $\mathbb{C}^{2}$ is the union of the graph with all vertical discs $\left\{e^{i \theta}\right\} \times \overline{\mathbb{D}}, \theta \in \mathbb{R}$. By the classical argument of Hartogs [18] any open Stein neighborhood of $K$ in $\mathbb{C}^{2}$ also contains the unit bidisc.

Problem 5.2. Characterize the bounded holomorphic functions on the disc $\mathbb{D}$ for which the closure of the graph admits a basis of open Stein neighborhoods in $\mathbb{C}^{2}$. 
The next two examples illustrate that Corollary 1.3 fails in general for images (as opposed to graphs) of maps. See however [10, Theorem 2.1].

EXAMPLE 5.3. This example was communicated to me by E. L. Stout (private communication, September 19, 2006):

For every $N>1$ there is an $\mathcal{A}(\mathbb{D})$-map $\overline{\mathbb{D}} \rightarrow \mathbb{C}^{N}$ whose image has no Stein neighborhood basis.

Let $E$ be a Cantor set of length zero contained in $b \mathbb{D}$; then $E$ is a peakinterpolation set for the disc algebra $\mathcal{A}(\mathbb{D})$. Let $\mathbb{B}$ denote the unit ball in $\mathbb{C}^{N}$ for some $N>1$. Choose a continuous map $f$ from $E$ onto the sphere $b \mathbb{B}$. There is a map $F=\left(F_{1}, \ldots, F_{N}\right): \overline{\mathbb{D}} \rightarrow \mathbb{C}^{N}$, satisfying $F_{j} \in \mathcal{A}(\mathbb{D})$ for each $j$, such that $\left.F\right|_{E}=f$ and $|F(z)|^{2}=\sum_{j=1}^{N}\left|F_{j}(z)\right|^{2}<1$ for all $z \in \overline{\mathbb{D}} \backslash E$ (Globevnik [15] and Stout [38]). Now let $D^{\prime}$ be a domain in $\mathbb{D}$ obtained by moving each of the open arcs of $b \mathbb{D} \backslash E$ in just a little, leaving the end points fixed; so $D^{\prime}$ is conformally a disc and $b D^{\prime} \cap b \mathbb{D}=E$. Then $F\left(\bar{D}^{\prime}\right)$ is a compact set consisting of the sphere $b \mathbb{B}$ together with a proper subset of $\mathbb{B}$, and hence it has no Stein neighborhood basis (any Stein neighborhood also contains the ball $\mathbb{B}$ ). It is necessary to pass to a smaller domain $D^{\prime} \subset \mathbb{D}$ because $F$ might take $\overline{\mathbb{D}}$ onto the ball $\overline{\mathbb{B}}$ which has a basis of Stein neighborhoods.

EXAMPLE 5.4. This example is a minor modification of the one which was communicated to me by J.-P. Rosay on April 6, 2004:

There exists a smooth $\left(\mathcal{C}^{\infty}\right)$ injective map $\Phi$ from the closed unit ball $\overline{\mathbb{B}}$ in $\mathbb{C}^{5}$ into $\mathbb{C}^{8}$, that is a holomorphic embedding of the open unit ball, such that $\Phi(\overline{\mathbb{B}})$ has no basis of Stein neighborhoods.

We proceed as follows. The set

$$
M=\left\{\left(z_{1}, \ldots, z_{5}\right) \in \overline{\mathbb{B}}: z_{1} z_{2} \cdots z_{5}=\sqrt{5}^{-5}\right\}
$$

is a real four dimensional submanifold of the boundary of $\mathbb{B}$ which is complex tangential to the sphere $b \mathbb{B}$ at each point. By Chaumat and Chollet ([5], [6]) every compact subset of $M$ is a peak interpolation set for $\mathcal{A}^{\infty}(\mathbb{B})$, the Fréchet algebra of functions holomorphic on the ball and smooth up to the boundary. Let $H$ be a closed Hartogs figure in $\mathbb{C}^{2}$ :

$$
\left(\zeta_{1}, \zeta_{2}\right) \in H \Longleftrightarrow\left(\left|\zeta_{1}\right| \leq 1,\left|\zeta_{2}\right| \leq \frac{1}{2}\right) \text { or }\left(\frac{1}{2} \leq\left|\zeta_{1}\right| \leq 1,\left|\zeta_{2}\right| \leq 1\right) .
$$

Let $H_{0}$ be a diffeomorphic copy of $H$ in $M$ (such exists by dimension reasons). Consider a smooth map $\varphi: \overline{\mathbb{B}} \rightarrow \mathbb{C}^{2}$ that is holomorphic on the open ball and whose restriction to $H_{0}$ is a diffeomeorphism from $H_{0}$ onto $H$. Also choose a function $h \in \mathcal{A}^{\infty}(\mathbb{B})$ that is zero on $H_{0}$ and that vanishes nowhere else on the closed unit ball. (Such $\phi$ and $h$ are obtained by appealing to [5], [6].) Define a map $\Phi: \overline{\mathbb{B}} \rightarrow \mathbb{C}^{8}$ by

$$
\Phi(z)=\Phi\left(z_{1}, \ldots, z_{5}\right)=\left(\phi(z), h(z), z_{1} h(z), \ldots, z_{5} h(z)\right) .
$$

It is easy to check that $\Phi$ is injective on $\bar{B}$, it is of maximum rank (immersion) at every point of $\overline{\mathbb{B}} \backslash H_{0}$, it maps $H_{0}$ onto $H \subset \mathbb{C}^{2} \times\{0\}^{6}$, and $\Phi(z) \notin \mathbb{C}^{2} \times\{0\}^{6}$ if $z \in \overline{\mathbb{B}} \backslash H_{0}$.

Since $\Phi(\overline{\mathbb{B}})$ contains the Hartogs figure $H \times\{0\}^{6}$, any open Stein neighborhood of it will also contain the unit bidisc in $\mathbb{C}^{2} \times\{0\}^{6}$; as this bidisc is not included in $\Phi(\overline{\mathbb{B}})$, the latter set has no basis of Stein neighborhoods. 
Problem 5.5. Let $K$ be a compact set with a Stein neighborhood basis in a complex manifold $S$. Assume that $f: K \rightarrow Y$ is a continuous map to a complex manifold $Y$ which is a uniform limit on $K$ of a sequence of holomorphic maps $f_{j}: V_{j} \rightarrow$ $Y$ defined in open neighborhoods of $K$.

Does the graph $G_{f}=\{(z, f(z)): z \in K\}$ admit a basis of open Stein neighboorhoods in $S \times Y$ ?

The answer is easily seen to be affirmative if $Y=\mathbb{C}^{N}$ and, by the embedding theorem, also if $Y$ is a Stein manifold. When $K$ is the closure of a strongly pseudoconvex domain the answer is given by Corollary 1.3. Another case of interest is the closure of a weakly pseudoconvex domain $D$ such that $K=\bar{D}$ admits a Stein neighborhood basis; the latter condition is necessary as is shown by the worm domain of Diederich and Fornæss [7].

Acknowledgements. The methods used in this paper are based on my joint works $[10,11]$ with Barbara Drinovec-Drnovšek whom I thank for her indirect contribution. I also wish to thank Debraj Chakrabarti and Laszlo Lempert for helpful remarks, Jean-Pierre Rosay and Edgar Lee Stout for kindly letting me include their examples in $\S 5$, and the referee for kindly explaining me the construction of Stein neighborhoods in Theorem 1.2 by using plurisubharmonic functions.

\section{REFERENCES}

[1] Calderón, A.-P., Lebesgue spaces of differentiable functions and distributions, Proc. Sympos. Pure Math., Vol. IV, pp. 33-49, American Mathematical Society, Providence, R.I., 1961.

[2] Campana, F., Peternell, T., Cycle spaces, Several complex variables, VII, pp. 319-349, Encyclopaedia Math. Sci., 74, Springer, Berlin, 1994.

[3] Cantor, M., Spaces of functions with asymptotic conditions on $\mathbb{R}^{n}$, Indiana Univ. Math. J., $24(1974 / 75)$, pp. 897-902.

[4] Catlin, D., A Newlander-Nirenberg theorem for manifolds with boundary, Michigan Math. J., 35 (1988), pp. 233-240.

[5] Chaumat, J., Сhollet, A.-M., Ensembles pics pour $A^{\infty}(D)$, Ann. Inst. Fourier (Grenoble), 29 (1979), pp. 171-200.

[6] Chaumat, J., Chollet, A.-M., Caractérisation et propriétés des ensembles localement pics de $A^{\infty}(D)$, Duke Math. J., 47 (1980), pp. 763-787.

[7] Diederich, K., Forness, J.-E., Pseudoconvex domains: an example with nontrivial Nebenhülle, Math. Ann., 225 (1977), pp. 275-292.

[8] Dineen, S., Complex analysis on infinite dimensional spaces, Springer, Berlin, 1999.

[9] Docquier, F., Grauert, H., Levisches Problem und Rungescher Satz für Teilgebiete Steinscher Mannigfaltigkeiten, Math. Ann., 140 (1960), pp. 94-123.

[10] Drinovec-Drnovšek, B., Forstnerič, F., Holomorphic curves in complex spaces, Duke Math. J., to appear. arXiv: math.CV/0604118

[11] Drinovec-Drnovšek, B., Forstnerič, F., Approximation of holomorphic mappings on strongly pseudoconvex domains, Forum Math., to appear. arXiv: math.CV/0607185

[12] Elüasson, H. I., Geometry of manifolds of maps, J. Diff. Geom., 1 (1967), pp. 169-194.

[13] Forstnerič, F., Prezelu, J., Oka's principle for holomorphic fiber bundles with sprays, Math. Ann., 317 (2000), pp. 117-154.

[14] Garnett, J. B., Bounded Analytic Functions, Academic Press, New York, 1981.

[15] Globevnik, J., The Rudin-Carleson theorem for vector-valued functions, Proc. Amer. Math. Soc., 53 (1975), pp. 250-252.

[16] Grauert, H., On Levi's problem and the imbedding of real-analytic manifolds, Ann. of Math., (2) 68 (1958), pp. 460-472.

[17] Gunning, R. C., Rossi, H., Analytic functions of several complex variables, Prentice-Hall, Englewood Cliffs, 1965.

[18] Hartogs, F., Zur Theorie der analytischen Funktionen mehrerer unabhängiger Veränderlichen, insbesondere über die Darstellung derselben durch Reihen, welche nach Potenzen einer Veränderlichen fortschreiten, Math. Ann., 62 (1906), pp. 1-88. 
[19] Heunemann, D., An approximation theorem and Oka's principle for holomorphic vector bundles which are continuous on the boundary of strictly pseudoconvex domains, Math. Nachr., 127 (1986), pp. 275-280.

[20] Heunemann, D., Theorem B for Stein manifolds with strictly pseudoconvex boundary, Math. Nachr., 128 (1986), pp. 87-101.

[21] Heunemann, D., Extension of the complex structure from Stein manifolds with strictly pseudoconvex boundary, Math. Nachr., 128 (1986), pp. 57-64.

[22] Hörmander, L., An Introduction to Complex Analysis in Several Variables, Third ed. North Holland, Amsterdam, 1990.

[23] Ivashrovich, S. M., Shevchishin, V. V., Deformations of noncompact complex curves, and envelopes of meromorphy of spheres (Russian), Mat. Sb., 189:9 (1998), pp. 23-60; English translation in Sb. Math., 189:9-10 (1998), pp. 1335-1359.

[24] Ivashrovich, S. M., Shevchishin, V. V., Structure of the moduli space in a neighborhood of a cusp-curve and meromorphic hulls, Invent. Math., 136 (1999), pp. 571-602.

[25] Krikorian, N., Differentiable structures on function spaces, Trans. Amer. Math. Soc., 171 (1972), pp. 67-82.

[26] Leiterer, J., Analytische Faserbündel mit stetigem Rand über streng-pseudokonvexen Gebieten, I: Math. Nachr., 71 (1976), pp. 329-344. II: Math. Nachr., 72 (1976), pp. 201-217.

[27] Leiterer, J., Theorem B für analytische Funktionen mit stetigen Randwerten, Beiträge zur Analysis, 8 (1976), pp. 95-102.

[28] Lempert, L., The Dolbeault complex in infinite dimensions: I, J. Amer. Math. Soc., 11 (1998), pp. $485-520$.

[29] Lempert, L., Holomorphic functions on (generalized) loop spaces, Math. Proc. R. Ir. Acad., 104A (2004), pp. 35-46.

[30] Lions, J.-L., Magenes, E., Non-Homogeneous Boundary Value Problems and Applications, Vol. I. Springer, New York, 1972.

[31] Ohsawa, T., Holomorphic embedding of compact s.p.c. manifolds into complex manifolds as real hypersurfaces, Differential geometry of submanifolds (Kyoto, 1984), pp. 64-76, Lecture Notes in Math., 1090, Springer, Berlin, 1984.

[32] Palais, R. S., Foundations of global non-linear analysis, W. A. Benjamin, Inc., New YorkAmsterdam, 1968

[33] Palais, R. S., Banach manifolds of fiber bundle sections, Actes du Congrès International des Mathématiciens (Nice, 1970), Tome 2, pp. 243-249. Gauthier-Villars, Paris, 1971.

[34] Penot, J.-P., Topologie faible sur des variétés de Banach, Application aux géodésiques des variétés de Sobolev. J. Diff. Geom., 9 (1974), pp. 141-168.

[35] Riddell, R. C., A note on Palais' axioms for section functors, Proc. Amer. Math. Soc., 25 (1970), pp. 808-810.

[36] Schneider, M., Tubenumgebungen Steinscher Räume, Manuscripta Math., 18 (1976), pp. 391397.

[37] Siu, J.-T., Every Stein subvariety admits a Stein neighborhood, Invent. Math., 38 (1976), pp. 89-100.

[38] Stout, E. L., On some restriction algebras, Function Algebras (Proc. Internat. Sympos. on Function Algebras, Tulane Univ., New Orleans, La., 1965), pp. 6-11, Scott-Foresman, Chicago, 1966. 